\title{
Corrigendum: Val66Met polymorphism of BDNF alters prodomain structure to induce neuronal growth cone retraction
}

\author{
Agustin Anastasia, Katrin Deinhardt, Moses V. Chao, Nathan E. Will, Krithi Irmady, Francis S. Lee, \\ Barbara L. Hempstead \& Clay Bracken
}

Nature Communications 4:2490 doi: 10.1038/ncomms3490 (2013); Published 18 Sep 2013; Updated 8 Apr 2014

The glutaraldehyde fixation method used in this Article was previously published by Dieni et al. to detect BDNF propeptide, and should have been cited in the first paragraph of the Results section as follows: 'However, glutaraldehyde fixation of proteins to the transfer membranes following sodium dodecyl sulphate polyacrylamide gel electrophoresis (SDS-PAGE) according to Dieni et al., and the use of a BDNF prodomain-specific monoclonal antibody previously characterized ${ }^{10}$, facilitated its detection in the mouse hippocampus as a $15.5-\mathrm{kDa}$ band (Fig. 1a), in agreement with the findings of Dieni et al.'.

Dieni, S. et al. BDNF and its pro-peptide are stored in presynaptic dense core vesicles in brain neurons. J. Cell. Biol. 196, 775-788 (2012). 